\title{
Phase retrieval, error reduction algorithm, and Fienup variants: a view from convex optimization
}

\author{
Heinz H. Bauschke \\ Department of Mathematics and Statistics, University of Guelph, Guelph, Ontario N1G 2W1, Canada \\ Patrick L. Combettes \\ Laboratoire Jacques-Louis Lions, Université Pierre et Marie Curie-Paris 6, 75005 Paris, France \\ D. Russell Luke \\ Institut für Numerische und Angewandte Mathematik, Universität Göttingen, 37083 Göttingen, Germany
}

Received September 25, 2001; revised manuscript received January 15, 2002; accepted January 15, 2002

\begin{abstract}
The phase retrieval problem is of paramount importance in various areas of applied physics and engineering. The state of the art for solving this problem in two dimensions relies heavily on the pioneering work of Gerchberg, Saxton, and Fienup. Despite the widespread use of the algorithms proposed by these three researchers, current mathematical theory cannot explain their remarkable success. Nevertheless, great insight can be gained into the behavior, the shortcomings, and the performance of these algorithms from their possible counterparts in convex optimization theory. An important step in this direction was made two decades ago when the error reduction algorithm was identified as a nonconvex alternating projection algorithm. Our purpose is to formulate the phase retrieval problem with mathematical care and to establish new connections between well-established numerical phase retrieval schemes and classical convex optimization methods. Specifically, it is shown that Fienup's basic input-output algorithm corresponds to Dykstra's algorithm and that Fienup's hybrid input-output algorithm can be viewed as an instance of the Douglas-Rachford algorithm. We provide a theoretical framework to better understand and, potentially, to improve existing phase recovery algorithms. (C) 2002 Optical Society of America
\end{abstract}

OCIS codes: $100.5070,100.3020,100.3010$

\section{INTRODUCTION}

The phase retrieval problem consists in estimating the phase of a complex-valued function from measurements of its modulus and additional a priori information. It is of fundamental importance in numerous areas of applied physics and engineering ${ }^{1-8}$ and has been studied for over 40 years (see Refs. 9-11 and the references therein). Historically, the roots of the problem can be traced back to 1892: In a letter to A. Michelson, Lord Rayleigh stated that the continuous phase retrieval problem in interferometry was, in general, impossible to solve without a priori information on the symmetry of the data. ${ }^{12}$

As in many inverse problems, a common formulation of the phase retrieval problem is to seek as a solution any function that is consistent with the measurements as well as with a priori constraints. The Gerchberg-Saxton algorithm, ${ }^{13}$ as well as its descendent in the form of the error reduction algorithm, ${ }^{14}$ was the first widely used numerical scheme to solve this type of problem. While its intrinsic mechanism is clear physically-it consists of alternating backsubstitutions of known information in the spatial and Fourier domains-it was not initially understood mathematically. In particular, failure of convergence and stagnation of the iterates away from solution points were observed from the outset but lacked a sound mathematical explanation. In the early 1980s, with the work of Youla and Webb ${ }^{15}$ and others, ${ }^{16-18}$ the application of Brègman's method of successive projections ${ }^{19}$ to the recovery of a signal described by convex constraints generated considerable interest in the signal recovery community; see Refs. 20-23 and the extensive lists of references therein. It was natural to seek to embed other iterative methods in this powerful projection framework. Thus the informal use of cyclic projections in the presence of nonconvex sets appears in several places in the literature. ${ }^{24-27}$ In Ref. 28, the error reduction algorithm (loosely called the Gerchberg-Saxton algorithm there) was revealed as such an algorithm, featuring a nonconvex magnitude constraint in the underlying signal space. This study (see also the follow-up paper ${ }^{29}$ ) gave insightful geometrical interpretations of the stagnation problem as well as of other aspects of the error reduction iterative procedure. A local convergence statement for the general nonconvex projection method was then proposed in Ref. 30 and followed in Ref. 31 by a more formal analysis based on the theory of multivalued projections (see also Ref. 32 for a tutorial review of these two papers, and Ref. 33 for further developments). Another approach to the convergence question was proposed in Refs. 34 and 35, based on the projection theory for convex sets. To extend 
this theory to the nonconvex setting, the authors require the projection operators to be single valued. ${ }^{36}$ Unfortunately, there is no known example of a nonconvex set for which the projection operator is single valued (see Remark 3.10). Indeed, the projections in the phase retrieval problem are inherently multivalued: In Ref. 38, the projection operator is precisely identified with the multivalued subdifferential of a related nonsmooth error metric. A smooth approximation to the projection operator is presented in Ref. 39 together with results on the local convergence of iterative methods for minimizing a corresponding smooth error metric.

In a series of papers ${ }^{40-42}$ that were unified and reviewed in his seminal 1982 paper, ${ }^{14}$ Fienup introduced a broad framework for iterative algorithms. Three main classes of algorithms were presented: error reduction, basic input-output (BIO), and hybrid input-output (HIO). Of the three classes, the last is the most widely used by practitioners. This work resulted in new applications in a wide range of imaging modalities. Furthermore, the error reduction and Fienup algorithms continue to constitute the central conceptual framework for phase retrieval algorithms.

While well-known in the field, the BIO and HIO algorithms lack a proper mathematical framework. The aim of this paper is to show that, just like the error reduction algorithm, the $\mathrm{BIO}$ and $\mathrm{HIO}$ algorithms also have powerful counterparts in the world of convex projection methods. Our discussion is somewhat more formal than what is usually found in the optics literature, as the level of sophistication of the algorithms requires great attention to details. As much as possible, we provide an intuitive and nontechnical discussion without sacrificing the mathematical rigor and precision necessary for a meaningful and constructive analysis of Fienup's algorithms. All technical proofs have been relegated to an appendix.

The paper is organized as follows. In Section 2, the phase retrieval problem is posed as a feasibility problem. Section 3 supplies the necessary review of projection theory, convex analysis, and fixed-point theory. The classical algorithms for solving the phase retrieval problem are presented in Section 4. Section 5 describes the correspondence between these algorithms and classical algorithms for solving the convex optimization problems: error reduction and alternating projections (Subsection 5.B); Fienup's BIO method and Dykstra's algorithm (Subsection 5.C), and Fienup's HIO method and DouglasRachford algorithm (Subsection 5.D). Concluding remarks are formulated in Section 6.

\section{PHASE RETRIEVAL AND FEASIBILITY}

In its general form, the signal recovery problem is to estimate the original form of a signal $x$ in a functional space $\mathcal{L}$ from the measurements of physically related signals and a priori information. ${ }^{21,22}$ In phase retrieval problems, the measurements consist of the modulus $m$ of the Fourier transform $\hat{x}$ of $x$. In other words, the imaging model is described by the relationship

$$
|\hat{x}|=m \text {, }
$$

and $x$ is commonly referred to as the object or the input of the imaging model. For instance, in optical interferometry astronomy, $x$ is the scattering amplitude of some medium. The measurement $m$ is a nonnegative function that is proportional to the modulus of the spatial coherence function (Section 7.4 in Ref. 43); that is, $m$ is proportional to the modulus of the Fourier transform of the scattering amplitude.

A general signal space that appropriately models the underlying physics is the complex Hilbert space

$$
\mathcal{L}=L^{2}\left[\mathrm{R}^{N}, \mathrm{C}\right] .
$$

Hence a signal $x$ in $\mathcal{L}$ is a square-integrable function mapping a continuous variable $t \in \mathbb{R}^{N}$ to a complex number $x(t) \in$ C. The set of signals that satisfy the Fourier domain constraint (1) is ${ }^{44}$

$$
M=\{y \in \mathcal{L}:|\hat{y}|=m \text { a.e. }\} .
$$

In addition to the imaging model, an important piece of information that is typically available in phase retrieval problems is that the support of $x$ is contained in some set $D \subset \mathrm{R}^{N}$. If we let $1_{E}$ denote the characteristic function of a set $E \subset \mathbb{R}^{N}$ and $C E$ be its complement, this object domain constraint confines $x$ to the set

$$
S=\left\{y \in \mathcal{L}: y \cdot 1_{C D}=0\right\}
$$

The phase retrieval problem can be posed as that of finding a function $x \in \mathcal{L}$ that satisfies these two constraints, namely,

$$
\text { find some } x \in S \cap M \text {. }
$$

This formulation exhibits the phase retrieval problem as a problem of finding a point in the intersection of constraint sets, i.e., a set-theoretic estimation problem in the sense of Ref. 20. In mathematics (especially in optimization), problems of this kind are called feasibility problems. In this paper, we shall restrict our attention to the case when problem (5) is consistent, i.e., $S \cap M \neq \varnothing$. It should be noted, however, that occurrences of inconsistent set-theoretic formulations in phase retrieval or other signal recovery problems are far from being academic, as a result of noisy data, measurement errors, or inaccurate a priori information. ${ }^{21,46-49}$ Several investigations have been devoted to analyzing and coping with this situation in convex problems. ${ }^{50-55}$

While the infinite-dimensional space $\mathcal{L}$ appears to be the most appropriate signal space to model the physics of the problem and to describe the subtle properties of the algorithms in their full generality, we shall also call attention to finite-dimensional versions of the results whenever these happen to differ from their infinitedimensional counterparts. The reason for this is that in most numerical applications, the signals are sampled on a finite grid and the algorithms are implemented on a digital computer. ${ }^{56}$ In this context, the underlying Hilbert space is a Euclidean space whose dimension is determined by the number of samples. 


\section{FUNDAMENTALS OF NUMERICAL THEORY}

Before discussing the most common and successful algorithms for solving the phase retrieval problem, we establish the mathematical definitions, properties, and results that constitute the theoretical foundation of projection algorithms. We begin with a few basic definitions.

\section{A. Distances, Projections, and Projectors}

\section{Distance}

As we shall deal with different Hilbert spaces, we assume in this section that

$\mathcal{H}$ is a general Hilbert space with inner product

$$
\langle\cdot, \cdot\rangle \text { and norm }\|\cdot\|: x \mapsto \sqrt{\langle x, x\rangle} \text {. }
$$

For instance, if $\mathcal{H}=\mathcal{L}$, then $\langle x, y\rangle=\int x \bar{y}$ for $x, y \in \mathcal{H}$. Or $\langle x, y\rangle=x^{\mathrm{T}} y$ in $\mathbb{R}^{N}$. The quantity $\|x\|^{2}$ is simply the energy of a signal $x \in \mathcal{H}$.

Definition 3.1 (distance). Suppose that $x \in \mathcal{H}$.

(i) If $y$ is a point in $\mathcal{H}$, then the distance from $x$ to $y$ is $d(x, y)=\|x-y\|$.

(ii) If $Y$ is a set in $\mathcal{H}$, then the distance from $x$ to $Y$ is $d(x, Y)=\inf _{y \in Y} d(x, y) .{ }^{58}$

As we show in the following example, the distance from a point to a set may not be attained.

Example 3.2. Let $\mathcal{H}=\mathbb{R}^{2}$ be the Euclidean plane.

(i) If $x=(2,0)$ and $Y=\{y \in \mathcal{H}:\|y\| \leqslant 1\}$ is the unit ball, then $d(x, Y)=1$ and the distance is attained at $y=(1,0) \in Y: d(x, Y)=d(x, y)$. Moreover, $y$ is the only point in $Y$ with this property-every other point in $Y$ is further from $x$ than $y$ is.

(ii) If $x=(2,0)$ and $Y=\{y \in \mathcal{H}:\|y\|<1\}$ is the open unit ball, then still $d(x, Y)=1$, yet there is no point $y \in Y$ with $d(x, y)=d(x, Y)$; the distance from $x$ to $Y$ is not attained.

(iii) If $x=(0,0)$ and $Y=\{y \in \mathcal{H}:\|y\|=1\}$ is the unit circle, then $d(x, Y)=1=d(x, y)$ for all $y \in Y$; the distance is attained at every point in $Y$.

The points at which the distance to a set is attained are of great importance and the subject of Subsection 3.A.2.

\section{Projection Operators (Projectors)}

Definition 3.3 (projection operator). Suppose that $Y$ is a set in $\mathcal{H}$. If $x \in \mathcal{H}$, then the set of points in $Y$ nearest to $x$, namely,

$$
\{y \in Y: d(x, y)=d(x, Y)\},
$$

is denoted $\Pi_{Y}(x)$ and called the projection of $x$ onto $Y$. The induced operator $\Pi_{Y}$ is called the projection operator or the projector onto $Y$.

An interpretation from signal processing helps at this point: If $Y$ contains the signals satisfying a certain property, then the signals in $\Pi_{Y}(x)$ are the closest signals to $x$ satisfying this property. It is crucial to realize that the output of a projector $\Pi_{Y}$ form subsets of $Y$. These may be empty, be reduced to a single element, or have more than one element: Revisiting Example 3.2 and borrowing its notation, we see that (i) $\Pi_{Y}(x)=\{(1,0)\}$, (ii) $\Pi_{Y}(x)$ $=\varnothing$, and (iii) $\Pi_{Y}(x)=Y$, respectively. To bring out this behavior clearly, we say that the projector is a multifunction or a multivalued map. ${ }^{59}$

Remark 3.4 (single-valued selections of projectors). Let $\Pi_{Y}$ be the projector onto a proximinal set $Y$, i.e., $\Pi_{Y}(x)$ $\neq \varnothing$ for all $x \in \mathcal{H}$. Then we shall denote by $P_{Y}$ a selection of $\Pi_{Y}$, i.e., $P_{Y}(x) \in \Pi_{Y}(x)$ for all $x \in \mathcal{H}$. $\quad P_{Y}$ is therefore a single-valued operator. When $Y$ is a Chebyshev set, i.e., $\Pi_{Y}(x)$ is a singleton for all $x \in \mathcal{H}$, then $\Pi_{Y}$ has a unique selection $P_{Y}$, which is itself called the projector onto $Y$. This always occurs when the set $Y$ is closed and convex (see Fact 3.9 in Subsection 3.B).

3. Preview: Projections for the Phase Retrieval Problem In the setting of the phase retrieval problem, the abstract Hilbert space $\mathcal{H}$ is simply the function space $\mathcal{L}$ introduced in Section 2. The most common approach for solving the phase retrieval problem is to enforce the known object domain and Fourier domain constraints in some alternating fashion. Thus, given a signal $x$, the support constraint is naturally enforced by setting $x$ equal to zero outside the given domain $D$ through the transformation $x \mapsto x$ - $1_{D}$. As we shall now see, this simple operation is actually a projection.

Example 3.5 (support constraint). Suppose that $D$ is a measurable ${ }^{60}$ set in $\mathbb{R}^{N}$, and fix $x \in \mathcal{L}$. Then the projection (recall the notation of Remark 3.4) of $x$ onto the set $S$ of Eq. (4) is

$$
P_{S}(x)=x \cdot 1_{D}
$$

The same observation is true for the image modulus constraint. Approaches to enforce it are described below; again, these operations turn out to be projections.

Example 3.6 (image modulus constraint). Let $m$ be a nonnegative function in $\mathcal{L}$, and fix $x \in \mathcal{L}$. Then $y \in \mathcal{L}$ belongs to the projection $\Pi_{M}(x)$ of $x$ onto the set $M$ of Eq. (3) if and only if it satisfies a.e.

$$
\hat{y}(\omega)= \begin{cases}m(\omega) \frac{\hat{x}(\omega)}{|\hat{x}(\omega)|} & \text { if } \hat{x}(\omega) \neq 0 \\ m(\omega) \exp [i \phi(\omega)] & \text { otherwise }\end{cases}
$$

for some measurable function $\phi: \mathbb{R}^{N} \rightarrow R$.

Example 3.6 shows that every function $y \in \Pi_{M}(x)$ satisfies

$$
d(\hat{x}(\omega), m(\omega) \mathrm{S})=d(\hat{x}(\omega), \hat{y}(\omega)) \quad \text { a.e. on } \mathbb{R}^{N},
$$

where $m(\omega) \mathrm{S}=\{u \in \mathrm{C}:|u|=m(\omega)\}$ denotes a circle in the complex plane with radius $m(\omega)$ and centered at the origin. The multivaluedness of the projection is now evident: Whenever $\hat{x}(\omega)=0$, any phase $\phi$ will work. Consequently, if the set $\left\{\omega \in \mathbb{R}^{N}: m(\omega) \neq 0\right.$ and $\left.\hat{x}(\omega)=0\right\}$ is sufficiently large, ${ }^{61}$ then $\Pi_{M}(x)$ contains infinitely many elements (see Ref. 39 and Example 3.15 in Subsection 3.B).

In practice, one picks the particular selection $y_{0} \in \Pi_{M}(x)$ corresponding to zero phase $\phi \equiv 0$ :

$$
\hat{y}_{0}(\omega)= \begin{cases}m(\omega) \frac{\hat{x}(\omega)}{|\hat{x}(\omega)|} & \text { if } \hat{x}(\omega) \neq 0 \\ m(\omega) & \text { otherwise }\end{cases}
$$


Analogous formulas hold if one considers a modulus constraint in the object domain (as in the original setup of the Gerchberg-Saxton algorithm for reconstructing phase from two intensity measurements; see Ref. 13).

\section{B. Convexity and Closedness}

In what follows, we assume that ${ }^{62}$

$\mathcal{H}$ is a real Hilbert space with inner product

$\langle\cdot, \cdot\rangle$ and induced norm $\|\cdot\|$.

Definition 3.7 (vector subspace and convex set). Suppose that $C$ is a nonempty set in $\mathcal{H}$. Then $C$ is a

(i) vector subspace if it contains the zero vector and if the line joining any two points in $C$ lies entirely in $C$ [algebraically, $\lambda c_{1}+(1-\lambda) c_{2} \in C$ whenever $c_{1}, c_{2} \in C$ and $\lambda \in R$,

(ii) convex set if the line segment joining any two points in $C$ lies entirely in $C$ (algebraically, $\lambda c_{1}+(1$ $-\lambda) c_{2} \in C$ whenever $c_{1}, c_{2} \in C$ and $\left.\lambda \in[0,1]\right)$.

Definition 3.8 (closed set). Suppose that $C$ is a set in $\mathcal{H}$. Then $C$ is closed if, whenever $\left(c_{n}\right)$ is a sequence in $C$ that converges to some point $c \in \mathcal{H}$, the limit point $c$ belongs necessarily to $C$.

Closedness is important for algorithmic purposes: One often wants the limit of a sequence to inherit good properties from the terms of the sequence. Closedness is certainly a necessary condition for the existence of projections. Indeed, a point $x$ in the closure (smallest closed superset) of $C$ but not in $C$ has no projection onto $C$. In finite-dimensional spaces, closedness is also sufficient to guarantee the existence of projections (e.g., Ref. 31); however, this is no longer true in infinite-dimensional spaces (see Example III.4.3.2.b in Ref. 63 for a counterexample).

In tandem with convexity, closedness guarantees that projections are extremely well behaved.

Fact 3.9 (projection onto a closed convex set). Suppose that $C$ is a nonempty closed convex set in $\mathcal{H}$. Then, for every $x \in \mathcal{H}$, the projection of $x$ onto $C$ is a singleton; moreover, the point ${ }^{64} P_{C}(x)$ is characterized by

$$
\begin{array}{r}
P_{C}(x) \in C, \quad\left\langle c-P_{C}(x), x-P_{C}(x)\right\rangle \leqslant 0 \\
\text { for all } c \in C .
\end{array}
$$

In addition, the projector $P_{C}$ satisfies

$$
\begin{aligned}
\left\|P_{C}(x)-P_{C}(y)\right\|^{2}+ & \left\|\left(I-P_{C}\right)(x)-\left(I-P_{C}\right)(y)\right\|^{2} \\
& \leqslant\|x-y\|^{2} \text { for all } x, y \in \mathcal{H} .
\end{aligned}
$$

Remark 3.10 (Chebyshev problem). Suppose that $C$ is a closed nonempty set in $\mathcal{H}$. If $C$ is convex, then Fact 3.9 states that the projector $\Pi_{C}=\left\{P_{C}\right\}$ is a single-valued map. The converse implication is the famous Chebyshev problem: if the projector $\Pi_{C}$ is a single-valued map, must the set $C$ be convex? The answer is affirmative in finite-dimensional spaces but remains open to date for the general Hilbert space case. If it turns out to be affirmative in general, then the results of Refs. 34 and 35 discussed in the Introduction (Section 1) are essentially void. The reader is referred to Chap. 12 in Ref. 65 for further information.
For the remainder of this section, we use the notation of Section 2. The following result is quite useful.

Proposition 3.11 (separable constraints and projections). Suppose that $(A(t))_{t \in \mathbb{R}^{N}}$ is a family of sets in C. Let $\mathcal{A}=\{a \in \mathcal{L}: a(t) \in A(t)$ a.e. $\}$.

(i) If each $A(t)$ is convex, then so is $\mathcal{A}$.

(ii) If each $A(t)$ is closed, then so is $\mathcal{A}$.

Theorem 3.12 (projection onto a separably closed convex constraint). Let $(A(t))_{t \in \mathbb{R}^{N}}$ be a family of closed convex sets in $\mathrm{C}$ such that $\mathcal{A}=\{a \in \mathcal{L}: a(t) \in A(t)$ a.e. $\}$ is nonempty. Assume that $t \mapsto A(t)$ is a measurable multifunction $^{66}$ from $\mathbb{R}^{N}$ to $\mathrm{C}$. Fix $x \in \mathcal{L}$. Then $y=P_{\mathcal{A}}(x)$ is given by $y(t)=P_{A(t)}(x(t))$ a.e.

Remark 3.13. In Theorem 3.12, the condition that $t \mapsto A(t)$ be measurable may be difficult to verify in practice. For our purpose, however, it is sufficient to work with the following criterion, taken from Section 14.A in Ref. 67:

If $\mu$ is a measurable function from $\mathbb{R}^{N}$ to $\mathrm{C}$ and $Z \subset \mathrm{C}$, then $A(t)=\mu(t) \cdot Z$ defines a measurable multifunction.

Note that there is no restriction whatsoever on the set $Z$.

Example 3.14 (object domain constraint). Let $x \in \mathcal{L}$. Then

(i) (support) $\mathcal{A}=\left\{y \in \mathcal{L}: y \cdot 1_{C D}=0\right\}$ is closed and convex, with $P_{\mathcal{A}}(x)=x \cdot 1_{D}$,

(ii) (real valuedness) $\mathcal{A}=\{y \in \mathcal{L}: y(t) \in \mathbb{R}$ a.e. $\}$ is closed and convex, with $P_{\mathcal{A}}(x)=\operatorname{Re}(x)$,

(iii) (nonnegativity) $\mathcal{A}=\{y \in \mathcal{L}: y(t)$ is real and nonnegative a.e. $\}$ is closed and convex (but not a vector subspace), with ${ }^{68} P_{\mathcal{A}}(x)=[\operatorname{Re}(x)]^{+}$.

Theorem 3.12 provides a convenient expression for the projection in the infinite-dimensional space $\mathcal{L}$ in terms of the finite-dimensional pointwise projections when the constraint is convex. Unfortunately, it is well-known that the phase retrieval problem involves nonconvex constraints, as the next example illustrates.

Example 3.15 (Fourier domain constraint is closed but not convex). For closedness, see the proof in Appendix A. Unless $m=0$ (in which case the Fourier modulus constraint encompasses only the zero function), the Fourier modulus constraint is never a convex set. To see this, pick $x \in M$. Then $-x \in M$; however, the convex combination $\frac{1}{2} x+\frac{1}{2}(-x)=0$ does not belong to $M$.

The next theorem states that certain nonconvex constraints can also be dealt with pointwise. This justifies the expression for the projection in Example 3.6.

Theorem 3.16 (projection onto a separably compact constraint). Let $(A(t))_{t \in \mathbb{R}^{N}}$ be a family of compact (i.e., closed and bounded) sets in $\mathrm{C}$ such that $\mathcal{A}=\{a$ $\in \mathcal{L}: a(t) \in A(t)$ a.e. $\}$ is nonempty. Assume that $t \mapsto A(t)$ is a measurable multifunction from $\mathbb{R}^{N}$ to $\mathrm{C}$. Then, for every $x \in \mathcal{L}, \Pi_{\mathcal{A}}(x) \neq \varnothing$; in fact, $y \in \Pi_{\mathcal{A}}(x)$ if and only if $y$ is measurable and $y(t) \in \Pi_{A(t)}(x(t))$ a.e.

While the phase retrieval problem (5) is not convex, some related problems that are convex can be found in the literature. 
(i) In the problem considered by Gerchberg in Ref. 69, the object domain constraint is again $x \cdot 1_{C D}=0$, and the Fourier domain constraint is $\hat{x} \cdot 1_{\Omega}=f$; i.e., the Fourier transform of $x$ (not just its modulus) on a domain $\Omega$ is a known function $f$. This constraint forms a convex set (actually an affine subspace, i.e., the translation of a vector subspace). Hence the resulting feasibility problem is convex (affine), which explains the good convergence properties of the alternating projection algorithm proposed by Gerchberg to solve it. This observation was made by Youla ${ }^{70}$ in the case of the Papoulis extrapolation algorithm for band-limited signals ${ }^{71}$ (this algorithm is identical to Gerchberg's, except that the roles played by the object and Fourier domains are interchanged).

(ii) In some problems, e.g., in holography, the Fourier domain constraint arises from the knowledge of the phase $\phi$ of the Fourier transform of $x$ rather than from its modulus. In Ref. 15, Youla and Webb observed that the phase constraint $\angle \hat{x}=\phi$ leads to a convex set (actually a convex cone). This fact was fully exploited in Ref. 17 (see also Ref. 29 and references therein).

(iii) In Ref. 15, Youla and Webb pointed out that the submodulus constraint $|\hat{x}| \leqslant m$ is convex. In most phase retrieval problems, this convexification of the exact constraint is too coarse, and it will typically produce poor results.

(iv) Convexity is an algebraic notion, which, by definition, depends on the choice of the underlying vector space structure. In Ref. 72, Çetin and Ansari exhibited an alternative (discrete) signal space in which the constraint $|\hat{x}|=m$ is convex. Unfortunately, this approach is not suitable for the phase retrieval problem, since the constraint $x \cdot 1_{C D}=0$ is no longer convex in this space.

Ultimately, the difficulty of the phase retrieval problem is caused by the lack of convexity of the Fourier domain constraint and the lack of good convex approximations to it.

\section{Some Fixed-Point Theory}

Throughout this subsection, we continue to make assumption (11).

Definition 3.17 (firm nonexpansivity and nonexpansivity). Suppose that $T$ is a map from $\mathcal{H}$ to $\mathcal{H}$. Then $T$ is firmly nonexpansive if

$$
\begin{aligned}
\|T(x)-T(y)\|^{2}+ & \|(I-T)(x)-(I-T)(y)\|^{2} \\
& \leqslant\|x-y\|^{2} \quad \text { for all } x, y \in \mathcal{H},
\end{aligned}
$$

and $T$ is nonexpansive if

$$
\|T(x)-T(y)\| \leqslant\|x-y\| \quad \text { for all } x, y \in \mathcal{H} .
$$

Fact 3.9 states that the projector onto a nonempty closed convex set is firmly nonexpansive. From the definition, it is immediate that every firmly nonexpansive map is nonexpansive. Firmly nonexpansive and nonexpansive maps are actually closely related.

Fact 3.18 (Theorem 12.1 in Ref. 73). Suppose that $T$ is a map from $\mathcal{H}$ to $\mathcal{H}$. Then the following are equivalent:

(i) $T$ is firmly nonexpansive,

(ii) $2 T-I$ is nonexpansive,

(iii) $T=\frac{1}{2} T+\frac{1}{2} I$ for some nonexpansive map $T$.
Many problems can be reduced to finding a fixed point of a nonexpansive mapping: The fixed-point set of a mapping $T$ from $\mathcal{H}$ to $\mathcal{H}$ is

$$
\operatorname{Fix} T=\{x \in \mathcal{H}: T(x)=x\}
$$

For example, the set of fixed points of a projector onto a closed convex set $C$ is just Fix $P_{C}=C$.

Fixed points are usually found as limit points of sequences. Discussing convergence in infinite-dimensional spaces requires care, because there exist distinct notions of convergence. The following concepts are appropriate in our present setting.

Definition 3.19 (norm and weak convergence). Suppose that $\left(x_{n}\right)$ is a sequence in $\mathcal{H}$ and that $x \in \mathcal{H}$. Then

(i) $\left(x_{n}\right)$ converges (in norm, or strongly) to $x$ if $\left\|x_{n}-x\right\| \rightarrow 0$ (in symbols, $x_{n} \rightarrow x$ ),

(ii) $\left(x_{n}\right)$ converges weakly to $x$ if $\left\langle x_{n}-x, y\right\rangle \rightarrow 0$ for all $y \in \mathcal{H}$ (in symbols $\left.x_{n} \rightarrow x\right)$.

Physically, $x_{n} \rightarrow x \in \mathcal{H}$ means that the energy of the residual signal $\left\|x_{n}-x\right\|^{2}$ becomes arbitrarily small as $n$ increases; on the other hand, $x_{n} \rightarrow x$ means only that any measurement of the residual signal that can be modeled by a linear operation from $\mathcal{H}$ to $\mathrm{R}$ becomes arbitrarily small.

It is easy to see that if a sequence converges in norm, then it does so weakly [indeed, if $x_{n} \rightarrow x$ and $y \in \mathcal{H}$, then, by the Cauchy-Schwarz inequality, $\left|\left\langle x_{n}-x, y\right\rangle\right|$ $\leqslant\left\|x_{n}-x\right\| \cdot\|y\| \rightarrow 0$ ]; in finite-dimensional spaces, the converse is true as well. However, in every infinitedimensional space, there exist sequences that converge weakly but not in norm (for instance, every orthonormal sequence converges weakly to zero but not in norm).

We shall see in Section 4 that it is desirable to have algorithms that find fixed points of nonexpansive mappings. The strong interest in firmly nonexpansive mappings stems from the ease of finding their fixed points by simple iteration.

Fact $3.20\left(\mathrm{Opial}^{74}\right)$. Suppose that $T$ is a firmly nonexpansive mapping from $\mathcal{H}$ to $\mathcal{H}$ with $\operatorname{Fix} T \neq \varnothing$. Then, for every $x \in \mathcal{H}$, the sequence $\left(T^{n} x\right)$ converges weakly to some point in Fix $T$.

Remark 3.21. An ingenious example by Genel and Lindenstrauss ${ }^{75}$ shows that it is not possible to strengthen the conclusion of Fact 3.20 to norm convergence. Also, if $T$ is nonexpansive (but not firmly), then $\left(T^{n} x\right)$ need not converge to a fixed point: Consider $T=-I$. Then Fix $T=\{0\}$, and $T^{n} x=(-1)^{n} x$ is not convergent, for all $x \neq 0$.

\section{CLASSICAL ALGORITHMS}

We now discuss three popular algorithms designed for solving the phase retrieval problem (5): Given a starting point $x_{0}$, each of these algorithms constructs a sequence $\left(x_{n}\right)$ of functions that in practice often converges to a solution of problem (5). The features common to all three algorithms are these: The construction of a function $x_{n+1}$ 
depends only on the predecessor $x_{n}$, and $x_{n+1}$ is found by applying the projection operators $P_{S}$ and $P_{M}$ in some fashion to $x_{n}$. Because of this, each algorithm is entirely characterized by its updating rule. ${ }^{76}$

We follow Fienup's framework. ${ }^{14}$ To bring out the results as clearly as possible, we assume that the object domain constraint is only a support constraint. In addition, for the sake of definiteness, $P_{M}$ designates the selection of the Fourier domain projector $\Pi_{M}$ defined through Eq. (10) (see Remark 3.4).

\section{A. Error Reduction Algorithm}

The error reduction algorithm updates a current iterate $x_{n}$ through $^{78}$

$$
x_{n+1}(t)=\left\{\begin{array}{ll}
\left(P_{M}\left(x_{n}\right)\right)(t) & \text { if } t \in D \\
0 & \text { otherwise }
\end{array} .\right.
$$

Hence $x_{n+1}=1_{D} \cdot P_{M}\left(x_{n}\right)$; equivalently, by Example 3.5 ,

$$
x_{n+1}=\left(P_{S} P_{M}\right)\left(x_{n}\right) .
$$

Local convergence results for this type and other types of nonconvex successive projection methods can be found in Ref. 31.

\section{B. Fienup's Basic Input-Output Algorithm}

The update $x_{n+1}$ in the BIO algorithm is obtained from $x_{n}$ by setting

$$
x_{n+1}(t)=\left\{\begin{array}{ll}
x_{n}(t) & \text { if } t \in D \\
x_{n}(t)-\left(P_{M}\left(x_{n}\right)\right)(t) & \text { otherwise }
\end{array} .\right.
$$

Note that $x_{n+1}=1_{D} \cdot x_{n}+1_{C D} \cdot\left[x_{n}-P_{M}\left(x_{n}\right)\right]=x_{n}$ - $\left(1-1_{D}\right) \cdot P_{M}\left(x_{n}\right)$, which we rewrite as

$$
x_{n+1}=\left(P_{S} P_{M}+I-P_{M}\right)\left(x_{n}\right) .
$$

\section{Fienup's Hybrid Input-Output Algorithm}

Given a parameter $\beta>0$, the HIO algorithm constructs the successor of $x_{n}$ through

$$
x_{n+1}(t)=\left\{\begin{array}{ll}
\left(P_{M}\left(x_{n}\right)\right)(t) & \text { if } t \in D \\
x_{n}(t)-\beta\left(P_{M}\left(x_{n}\right)\right)(t) & \text { otherwise }
\end{array} .\right.
$$

For clarity, we henceforth set $\beta=1$; in practice, values of $\beta$ different from 1 are important, as they may result in better performance., 3 The recursion (21) now becomes

$$
\begin{aligned}
x_{n+1} & =1_{D} \cdot P_{M}\left(x_{n}\right)+1_{C D} \cdot\left[x_{n}-P_{M}\left(x_{n}\right)\right] \\
& =1_{D} \cdot P_{M}\left(x_{n}\right)+\left(1-1_{D}\right) \cdot\left[x_{n}-P_{M}\left(x_{n}\right)\right] \\
& =1_{D} \cdot\left[2 P_{M}\left(x_{n}\right)-x_{n}\right]+x_{n}-P_{M}\left(x_{n}\right) \\
& =\left(P_{S}\left(2 P_{M}-I\right)+\left(I-P_{M}\right)\right)\left(x_{n}\right) .
\end{aligned}
$$

Note that this can also be written as

$$
x_{n+1}=\left(P_{S} P_{M}+\left(I-P_{S}\right)\left(I-P_{M}\right)\right)\left(x_{n}\right),
$$

because the projector onto a closed vector space is linear.

Remark 4.1. The description of the $\mathrm{HlO}$ algorithm in Ref. 14, specialized to our setting, actually reads as ${ }^{79}$

$$
\begin{aligned}
& x_{n+1}(t) \\
& =\left\{\begin{array}{ll}
\left(P_{M}\left(x_{n}\right)\right)(t) & \text { if } t \in D \text { or }\left(P_{M}\left(x_{n}\right)\right)(t)=0 \\
x_{n}(t)-\left(P_{M}\left(x_{n}\right)\right)(t) & \text { otherwise }
\end{array} .\right.
\end{aligned}
$$

The updates differ precisely at points $t$ that belong to $\complement D$ and that satisfy $\left(P_{M}\left(x_{n}\right)\right)(t)=0$. However, it is not easy to determine which formulation is used in the community, as the papers that we are aware of are not specific on this question. Notable exceptions are Refs. 80-82, which use the formulation that we shall employ, ${ }^{83}$ and Ref. 84, which uses the literal definition. We should note that the main results of this paper would remain essentially unchanged if we followed the literal definition. Interestingly, the same issue formally arises for the error reduction algorithm and for the BIO algorithm; however, a careful inspection reveals that this does not lead to different algorithms!

\section{MAIN CONNECTIONS WITH CONVEX OPTIMIZATION ALGORITHMS}

We are now ready to establish the correspondence between classical algorithms for solving problem (5) and their counterparts for solving a two-set convex feasibility problem. Throughout this section, the standing assumption is that

$A$ and $B$ are two nonempty closed convex sets in a real Hilbert space $\mathcal{H}$.

\section{A. Convex Feasibility Problem}

The convex feasibility problem associated with $A$ and $B$ is to

$$
\text { find some } \quad x \in A \cap B .
$$

Note the similarity between problem (25) and the formulation (5) of the phase retrieval problem as a feasibility problem. However, problem (5) is not a convex feasibility problem, as the image modulus constraint is not convex (Example 3.15).

We now revisit the three classical algorithms for solving the phase retrieval problem described above. It will turn out that each algorithm corresponds to a classical algorithm for solving problem (25). ${ }^{85}$

\section{B. Error Reduction Algorithm and Projections onto Convex Sets}

The method of alternating projections onto convex sets (POCS) generates, for the present setting of two constraints, sequences $\left(a_{n}\right)$ and $\left(b_{n}\right)$ as follows: Pick an arbitrary starting point $a_{0} \in \mathcal{H}$. Then update for $n \geqslant 0$ through

$$
b_{n}=P_{B}\left(a_{n}\right), \quad a_{n+1}=P_{A}\left(b_{n}\right) .
$$

This process is depicted in Fig. 1.

The following basic result shows that the POCS method does find a solution of problem (25).

Fact 5.1 (Brègman $^{19}$ ). Suppose that $A \cap B \neq \varnothing$. Then both sequences $\left(a_{n}\right)$ and $\left(b_{n}\right)$ in Eq. (26) converge weakly to a point in $A \cap B$.

Remark 5.2. Although we do not go into details, we mention in passing two possible paths to proving Fact 5.1. The first approach is fixed-point theoretic and consists of 


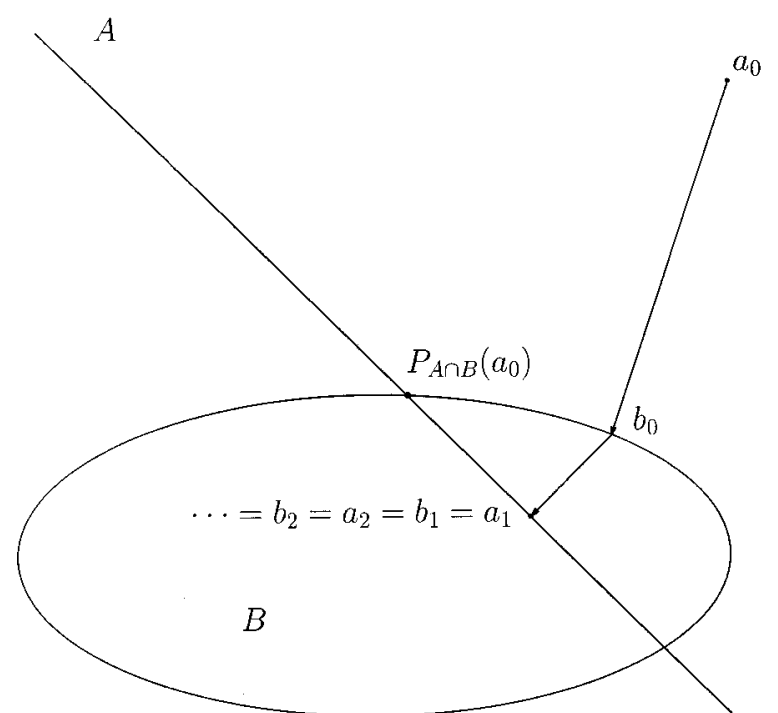

Fig. 1. POCS algorithm. The initial point $a_{0}$ is projected onto $B$ and then onto $A$. The point $a_{1}$ thus obtained belongs to both sets, and the algorithm therefore converges in two steps. Note that the solution $a_{1}$ is not the projection of $a_{0}$ onto $A \cap B$.

showing that (1) $\operatorname{Fix}\left(P_{A} P_{B}\right)=A \cap B$ and (2) iterating the composition $P_{A} P_{B}$ produces-analogously to Fact 3.20 -fixed points. ${ }^{89}$ The second approach is more elementary and builds on the notion of Fejér monotonicity; see Refs. 91-93.

Observation 5.3 (error reduction algorithm as a nonconvex POCS algorithm). Replace the set $A$ with the (convex) object domain constraint set $S$ and the set $B$ with the (nonconvex) Fourier domain constraint set $M$. Then the sequence $\left(a_{n}\right)$ generated by Eq. (26) corresponds to the sequence $\left(x_{n}\right)$ generated by the error reduction algorithm (18). This connection was established by Levi and Stark $^{28}$ in 1984.

\section{Fienup's Basic Input-Output Algorithm and Dykstra's Algorithm}

Dykstra's algorithm was first developed for closed convex cones in Ref. 94 and subsequently generalized to closed convex sets in Ref. 95. For two closed convex sets $A$ and $B$, it produces four sequences $\left(a_{n}\right),\left(b_{n}\right),\left(p_{n}\right)$, and $\left(q_{n}\right)$ as follows (see Fig. 2). Fix a starting point $a_{0}$, set $q_{-1}$ $=0=p_{0}$, and update for $n \geqslant 0$ through

$$
\begin{aligned}
b_{n} & =P_{B}\left(a_{n}+q_{n-1}\right), \\
q_{n} & =\left(I-P_{B}\right)\left(a_{n}+q_{n-1}\right)=a_{n}+q_{n-1}-b_{n}, \\
a_{n+1} & =P_{A}\left(b_{n}+p_{n}\right), \\
p_{n+1} & =\left(I-P_{A}\right)\left(b_{n}+p_{n}\right)=b_{n}+p_{n}-a_{n+1} .
\end{aligned}
$$

Clearly, Dykstra's algorithm is more involved than POCS and is more demanding in terms of storage. However, its convergence properties are superior in the sense that (1) it converges in norm and (2) it provides a welldefined limit point, namely, the feasible signal that lies closest to the starting point. ${ }^{96}$
Fact 5.4 (Boyle-Dykstra $\left.{ }^{95}\right)$. Suppose that $A \cap B$ $\neq \varnothing$. Then both sequences $\left(a_{n}\right)$ and $\left(b_{n}\right)$ in Eq. (27) converge in norm to $P_{A \cap B}\left(a_{0}\right)$, the point in $A \cap B$ closest to $a_{0}$.

Fact 5.4 is quite remarkable because the sequences converge in norm, and their limit is explicitly identified as the nearest feasible point to the starting point. This explains the popularity of Dykstra's algorithm in approximation theory, where this method is well understood and many extensions have been found; see, for instance, Refs. 98-102. For applications of Dykstra's algorithm to signal recovery, see Ref. 103.

For the rest of this subsection, we assume additionally that $A$ is a closed vector space. Then $\left(p_{n}\right)$ lies entirely in $A^{\perp}$, the orthogonal complement of $A$, and the computation of $a_{n+1}$ becomes $a_{n+1}=P_{A} b_{n}+P_{A} p_{n}=P_{A} b_{n}$. Thus the sequence $\left(p_{n}\right)$ is not needed, and Dykstra's algorithm simplifies to

$$
\begin{aligned}
b_{n} & =P_{B}\left(a_{n}+q_{n-1}\right), \\
q_{n} & =\left(I-P_{B}\right)\left(a_{n}+q_{n-1}\right), \\
a_{n+1} & =P_{A}\left(b_{n}\right) .
\end{aligned}
$$

Hence

$$
\begin{aligned}
a_{n+1}+q_{n} & =\left(P_{A} P_{B}+I-P_{B}\right)\left(a_{n}+q_{n-1}\right) \\
& =\left(P_{A} P_{B}+I-P_{B}\right)^{n+1}\left(a_{0}\right) .
\end{aligned}
$$

The next observation appears to be new; it establishes the correspondence between the BIO and Dykstra's algorithm.

Observation 5.5 (BIO algorithm as a nonconvex Dykstra algorithm). Replace the set $A$ with the (convex) object domain constraint set $S$ and the set $B$ with the (noncon-

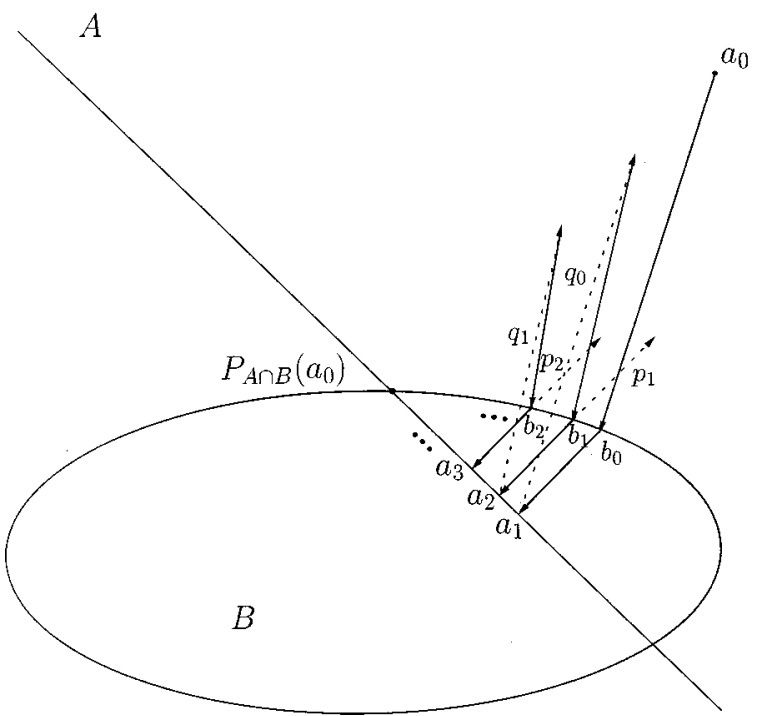

Fig. 2. Dykstra's algorithm. The first two steps of this algorithm are identical to those of the POCS algorithm (Fig. 1). Here, however, although $a_{1} \in A \cap B$, the algorithm does not reach convergence at this point, since the outward-pointing normal $q_{0}$ pulls the vector $a_{1}+q_{0}$ out of $B$ before it is projected onto $B$. Through this process, two infinite sequences $\left(a_{n}\right)$ and $\left(b_{n}\right)$ are generated that converge to $P_{A \cap B}\left(a_{0}\right)$. Note that since $A$ is an affine subspace in this example, $p_{n} \perp A$ and, therefore, $a_{n+1}$ $=P_{A}\left(b_{n}\right)$. 


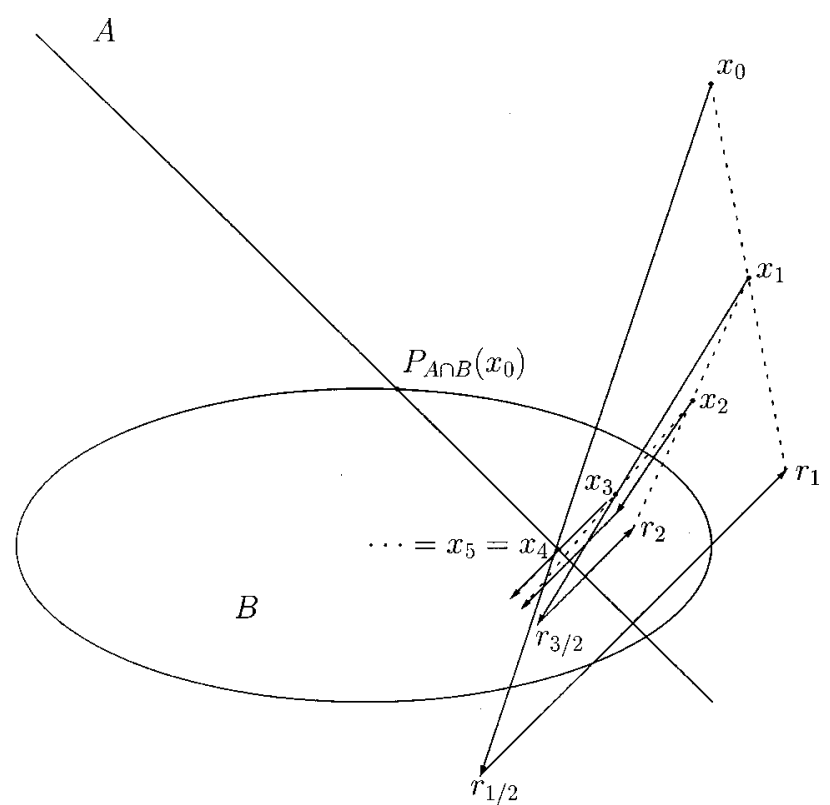

Fig. 3. Douglas-Rachford algorithm. The update equation (32) is executed as follows: One first computes the reflection $r_{n+(1 / 2)}$ of $x_{n}$ with respect to $B$ and then the reflection $r_{n+1}$ of $r_{n+(1 / 2)}$ with respect to $A$. The update $x_{n+1}$ is the midpoint of the segment between $x_{n}$ and $r_{n+1}$. In this example, the algorithm converges in four iterations, since $x_{4} \in A \cap B$.

vex) Fourier domain constraint set $M$. Then the sequence $\left(a_{n}+q_{n-1}\right)$ generated by Eq. (29) corresponds to the sequence $\left(x_{n}\right)$ generated by Fienup's BIO algorithm (20).

Remark 5.6. Even when $A \cap B \neq \varnothing$, it is possible that the sequences $\left(p_{n}\right)$ and $\left(q_{n}\right)$ generated by Dykstra's algorithm (in its general form) are both unbounded; see Ref. 101. This suggests that the pertinent sequence to monitor in Fienup's BIO algorithm is $\left(P_{M}\left(x_{n}\right)\right)$ rather than $\left(x_{n}\right)$.

\section{Fienup's Hybrid Input-Output Algorithm and} Douglas-Rachford Algorithm

When specialized to the convex feasibility problem (25), the Douglas-Rachford algorithm ${ }^{104}$ generates a sequence $\left(x_{n}\right)$, from an arbitrary starting point $x_{0}$, by

$$
x_{n+1}=\left(P_{A}\left(2 P_{B}-I\right)+\left(I-P_{B}\right)\right)\left(x_{n}\right) .
$$

For brevity, we set

$$
T=P_{A}\left(2 P_{B}-I\right)+\left(I-P_{B}\right) .
$$

If $A$ is a closed vector space, then $T$ can be written more symmetrically as $T=P_{A} P_{B}+\left(I-P_{A}\right)\left(I-P_{B}\right)$. Now let $R_{A}=2 P_{A}-I$ be the reflector with respect to $A$, and define $R_{B}$ likewise. The following proposition gives an alternative description of the Douglas-Rachford algorithm that lends itself to a simple geometrical interpretation (see Fig. 3).

Proposition 5.7. The mapping $T$ in Eq. (31) can be written as $T=\left(R_{A} R_{B}+I\right) / 2$. Hence Eq. (30) is equivalent to

$$
x_{n+1}=\frac{1}{2}\left(R_{A} R_{B}+I\right)\left(x_{n}\right) .
$$

The next two basic results on the Douglas-Rachford iteration are due to Lions and Mercier ${ }^{106}$; see also Refs. 87 and 88. We include some proofs in Appendix A, as they appear to be simpler than those found in the literature.

Fact 5.8. The mapping $T$ in Eq. (31) is firmly nonexpansive.

Fact 5.9 (Lions-Mercier ${ }^{106}$ ). Suppose that $A \cap B$ $\neq \varnothing$. Then the sequence $\left(x_{n}\right)$ generated by Eq. (32) converges weakly to some point $x \in \mathrm{Fix} T$, and $P_{B}(x)$ $\in A \cap B$. Moreover, the sequence $\left(P_{B}\left(x_{n}\right)\right)$ is bounded, and every weak cluster point ${ }^{107}$ of $\left(P_{B}\left(x_{n}\right)\right)$ lies in $A \cap B$. If $\mathcal{H}$ is finite dimensional, then $x_{n} \rightarrow x$ and $P_{B}\left(x_{n}\right)$ $\rightarrow P_{B}(x) \in A \cap B$.

The following connection, which identifies the HIO algorithm with the Douglas-Rachford algorithm, does not seem to have been drawn elsewhere.

Observation 5.10 (HIO algorithm as a nonconvex Douglas-Rachford algorithm). Replace the set $A$ with the (convex) object domain constraint set $S$ and the set $B$ with the (nonconvex) Fourier domain constraint set $M$. Then the sequence generated by the Douglas-Rachford algorithm (30) corresponds to the sequence generated by the HIO algorithm (22). ${ }^{108}$

\section{CONCLUDING REMARKS}

The contribution of this paper is twofold. First, an analysis of the phase retrieval problem has been carried out in the mathematical context of multivalued projection operators. This analysis provides rigorous and easily verifiable criteria for calculating projections. Second, new connections have been established between some classical phase retrieval methods and some standard convex optimization algorithms.

While the mathematical theory remains unable to completely analyze the convergence behavior of these algorithms in nonconvex settings, the analogies drawn here open the door for experimentation with variations that are well understood in convex settings. We believe that the convex-analytical viewpoint adopted in this paper can be exploited further in order to develop alternative phase retrieval schemes.

\section{APPENDIX A: PROOFS}

Proof of Example 3.5. See Example 3.14 for a rigorous proof.

Proof of Example 3.6. This is a sketch of the proof; see Theorem 4.2 in Ref. 39 for full details. Fix $x \in \mathcal{L}$. Because the Fourier transform is unitary, it follows that $\widehat{\Pi_{M}(x)}=\Pi_{\mathcal{A}}(\hat{x})$. In turn, by Theorem 3.16 , the projection onto $\mathcal{A}$ can be found separably, provided that the selection is measurable. But the projection onto the circle in C is easy: Radially scale the point, and observe the multivaluedness at the origin. The step from the measurable selection to the measurable phase $\phi$ requires a measure-theoretical argument; see the proof of Theorem 4.2 in Ref. 39.

Proof of Fact 3.9. This is part of the folklore. See, for instance, Lemma 1.1 in Ref. 109.

Proof of Proposition 3.11. Part (i) is easily verified. (ii) Let $\left(a_{n}\right)$ be a sequence in $\mathcal{A}$ converging to some 
$z \in \mathcal{L}$. A result by Riesz (see Theorem 12.6 in Ref. 45 or Theorem 2.8.2 in Ref. 110) implies that there exists a subsequence $\left(a_{k_{n}}\right)$ of $\left(a_{n}\right)$ that converges to $z$ a.e. Since $\left(a_{k_{n}}(t)\right)_{n}$ lies in the closed set $A(t)$ a.e., it follows that $z(t) \in A(t)$ a.e. Consequently, $\mathcal{A}$ is closed.

Proof of Theorem 3.12. Since each set $A(t)$ is closed convex and nonempty, the function $y: t \mapsto P_{A(t)}(x(t))$ is well defined. By Corollary 8.2.13(3) in Ref. 59 [see also Exercise 14.17.(b) in Ref. 67], $y$ is measurable. Pick an arbitrary $a \in \mathcal{A}$. Then $|x(t)-y(t)| \leqslant|x(t)-a(t)|$ a.e. Squaring and integrating yield $y \in \mathcal{L}$, and hence $y \in \Pi_{\mathcal{A}}(x)$. Since $\mathcal{A}$ is closed and convex by Proposition $3.11, \Pi_{\mathcal{A}}(x)$ is a singleton (Fact 3.9 ), which we write as $y=P_{\mathcal{A}}(x)$.

Proof of Example 3.14. We first define $A(t)$, depending on the case considered: (i) $A(t)=\mathrm{C}$ if $t \in D$ and $A(t)$ $=\{0\}$ otherwise, (ii) $A(t) \equiv \mathbb{R}$, and (iii) $A(t) \equiv \mathbb{R}_{+}$. Now let $\mathcal{A}$ be as in Theorem 3.12. Note that $\mathcal{A}$ is nonempty, as it contains the zero function. Next, observe that in each case, we can write $A(t)=\mu(t) \cdot Z$, where $\mu: \mathbb{R}^{N} \rightarrow \mathrm{C}$ is measurable and $Z \subset \mathrm{C}$ : Indeed, (i) $Z=\mathrm{C}$ and $\mu=1_{D}$, (ii) $Z=\mathrm{R}$ and $\mu \equiv 1$, and (iii) $Z=\mathrm{R}_{+}$and $\mu \equiv 1$. In view of Remark 3.13, $t \mapsto A(t)$ is a measurable multifunction in each case. The result now follows from Theorem 3.12 .

Proof of Theorem 3.16. Since $A$ is a measurable multifunction, the function $\mathbb{R}^{N} \rightarrow \mathbb{R}_{+}: \quad t \mapsto d(\rho, A(t))$ is measurable for every $\rho \in \mathbb{C}$ [Corollary 8.2.13(2) in Ref. 59 and Theorem 14.3 in Ref. 67]. On the other hand, the function $\mathrm{C} \rightarrow \mathbb{R}_{+}: \quad \rho \mapsto d(\rho, A(t))$ is continuous (even nonexpansive) for every $t \in \mathbb{R}^{N}$. Altogether, the function $\mathbb{R}^{N} \times \mathrm{C} \rightarrow \mathbb{R}_{+}: \quad(t, \rho) \mapsto d(\rho, A(t))$ is a Carathéodory function; see Definition 4.49 in Ref. 45. Now $\mathcal{A} \neq \varnothing$; hence $A$ is nonempty valued. By Theorem 17.5 in Ref. 45 , the multifunction $A$ is weakly measurable. Now fix $x \in \mathcal{L}$, and let $f: \mathbb{R}^{N} \times \mathrm{C} \rightarrow \mathbb{R}: \quad(t, \rho) \mapsto-|x(t)-\rho|$. Then $f(t, \rho)$ is measurable in $t$ and continuous in $\rho$; thus $f$ is also a Carathéodory function. The measurable maximum theorem (Theorem 17.18 in Ref. 45) yields that (1) $t \mapsto d(x(t), A(t))$ is a measurable function, (2) $t \mapsto \Pi_{A(t)}(x(t))$ is a measurable multifunction with nonempty compact values, and (3) there exists a measurable selection $z(t) \in \Pi_{A(t)}(x(t))$. Clearly, $z \in \Pi_{\mathcal{A}}(x)$. (This first part of the proof can also be contemplated from a higher perspective; see Section 8.2 in Ref. 59.) It remains to prove the equivalence concerning $\Pi_{\mathcal{A}}(x)$. The "if" part is clear. We now suppose to the contrary that the "only if" part is false. Then there exists a measurable function $y \in \Pi_{A}(x)$ such that the set $E=\{t$ $\left.\in \mathbb{R}^{N}:|x(t)-y(t)|>d(x(t), A(t))\right\}$ has strictly positive measure. But then

$$
\begin{aligned}
d^{2}(x, \mathcal{A}) & =\|x-y\|^{2} \\
& =\int_{E}|x(t)-y(t)|^{2} \mathrm{~d} t+\int_{C E}|x(t)-y(t)|^{2} \mathrm{~d} t \\
& >\int_{E}|x(t)-z(t)|^{2} \mathrm{~d} t+\int_{C E}|x(t)-z(t)|^{2} \mathrm{~d} t \\
& =\|x-z\|^{2}=d^{2}(x, \mathcal{A}),
\end{aligned}
$$

which is absurd.
Proof of Example 3.15. Recall that $m \in \mathcal{L}$ is the prescribed nonnegative modulus function. The set of all functions satisfying the image modulus constraint is $M=\{z \in \mathcal{L}:|\hat{z}|=m\}$. Note that $M \neq \varnothing$, since $m \in M$. Let $\mathrm{S}$ be the closed unit circle in $\mathrm{C}$, and set $A(\omega)=m(\omega)$. S for all $\omega \in \mathbb{R}^{N}$. The multifunction $\omega \mapsto A(\omega)$ is compact valued, nonempty valued, and measurable (Remark 3.13). Now let $\mathcal{A}=\{z \in \mathcal{L}: z(\omega)$ $\in A(\omega)$ a.e. $\}$ Then $\mathcal{A}$ is closed by Proposition 3.11(ii). Consequently, as the preimage of $\mathcal{A}$ under a continuous operator, namely, the Fourier transform, the set $M$ is closed. $^{111}$

Proof of Proposition 5.7. By simple expansion, we obtain

$$
\begin{aligned}
R_{A} R_{B} & =\left(2 P_{A}-I\right)\left(2 P_{B}-I\right) \\
& =2 P_{A}\left(2 P_{B}-I\right)+2\left(I-P_{B}\right)-I .
\end{aligned}
$$

Hence Eq. (31) yields $T=\frac{1}{2}\left(R_{A} R_{B}+I\right)$.

Proof of Fact 5.8. (See also Proposition 2 in Ref. 106 and Corollary 4.1 in Ref. 88.) Because $P_{A}$ and $P_{B}$ are projectors onto convex sets, they are firmly nonexpansive (Fact 3.9). Thus, by Fact 3.18, $R_{A}$ and $R_{B}$ are nonexpansive. Hence the composition $R_{A} R_{B}$ is nonexpansive, which, in turn, implies (Fact 3.18 again) that $\frac{1}{2}\left(R_{A} R_{B}\right.$ $+I$ ) is firmly nonexpansive. In view of Proposition 5.7, the proof is complete.

Fact $A 1 . \quad P_{B}($ Fix $T)=A \cap B \subset$ Fix $T$.

Proof. Fix an arbitrary $x \in \mathcal{H}$. Write $x=b+q$, where $b=P_{B}(x)$ and $q=x-b$. Then $b=P_{B}(x)$ $=P_{B}(b+q)$. The result follows from the equivalences $\quad x=T(x) \Leftrightarrow x=P_{A}\left(2 P_{B}-I\right)(x)+\left(I-P_{B}\right)(x)$ $\Leftrightarrow b+q=P_{A}(2 b-(b+q))+b+q-b \Leftrightarrow b$ $=P_{A}(b-q)$.

Proof of Fact 5.9. [See also Theorem 1(iii) and Remark 7 in Ref. 106 and Corollary 6.1 in Refs. 88 and 112.] By Fact A1, Fix $T$ contains $A \cap B \neq \varnothing$. In view of Fact 3.20 , the sequence $\left(x_{n}\right)=\left(T^{n}\left(x_{0}\right)\right)$ converges weakly to some fixed point $x$ of $T$. Since $P_{B}$ is nonexpansive and $\left(x_{n}\right)$ is bounded, it follows that $\left(P_{B}\left(x_{n}\right)\right)$ is bounded. Since $T$ is firmly nonexpansive, $\left\|x_{n}-x\right\|^{2} \geqslant\left\|x_{n+1}-x\right\|^{2}$ $+\left\|x_{n}-x_{n+1}\right\|^{2}$, which implies (after telescoping)

$$
0 \leftarrow x_{n}-x_{n+1}=P_{B}\left(x_{n}\right)-P_{A}\left(2 P_{B}\left(x_{n}\right)-x_{n}\right) .
$$

Hence weak cluster points of $\left(P_{B}\left(x_{n}\right)\right)$ must lie in $A \cap B$. Finally, if $\mathcal{H}$ is finite dimensional, then $P_{B}\left(x_{n}\right) \rightarrow P_{B}(x)$ $=P_{A}\left(2 P_{B}(x)-x\right) \in A$.

\section{ACKNOWLEDGMENTS}

This work was inspired by the workshop organized by John Spence on New Approaches to the Phase Problem for Non-Periodic Objects at the Lawrence Berkeley National Laboratory, May 17-19, 2001. We warmly thank Laurence Marks for many fruitful discussions and John Spence for making our participation in the workshop possible. We also express our gratitude to the three anonymous referees for their valuable remarks.

The work of H. H. Bauschke was supported by the Natural Sciences and Engineering Research Council of Canada, and the work of P. L. Combettes was partially 
supported by the National Science Foundation under grant MIP-9705504.

E-mails: bauschke@cecm.sfu.ca, plc@math.jussieu.fr, luke@math.uni-goettingen.de.

\section{REFERENCES AND NOTES}

1. A. Barty, D. Paganin, and K. Nugent, "Phase retrieval in Lorentz microscopy," Exp. Methods Phys. Sci. 36, 137-166 (2001).

2. J.-F. Brun, D. de Sousa Meneses, B. Rousseau, and P. Echegut, "Dispersion relations and phase retrieval in infrared reflection spectra analysis," Appl. Spectrosc. 55, 774780 (2001).

3. J. C. Dainty and J. R. Fienup, "Phase retrieval and image reconstruction for astronomy," in Image Recovery: Theory and Application, H. Stark, ed. (Academic, Orlando, Fla., 1987), pp. 231-275.

4. L. D. Marks, W. Sinkler, and E. Landree, "A feasible set approach to the crystallographic phase problem," Acta Crystallogr. Sect. A 55, 601-612 (1999)

5. R. P. Millane, "Phase retrieval in crystallography and optics," J. Opt. Soc. Am. A 7, 394-411 (1990).

6. P. Jaming, "Phase retrieval techniques for radar ambiguity problems," J. Fourier Anal. Appl. 5, 309-329 (1999).

7. W. O. Saxton, Computer Techniques for Image Processing in Electron Microscopy (Academic, New York, 1978).

8. L. S. Taylor, "The phase retrieval problem," IEEE Trans. Antennas Propag. AP-29, 386-391 (1981)

9. P. Roman and A. S. Marathay, "Analyticity and phase retrieval," Nuovo Cimento 30, 1452-1464 (1963).

10. A. Walther, "The question of phase retrieval in optics," Opt. Acta 10, 41-49 (1963).

11. E. Wolf, "Is a complete determination of the energy spectrum of light possible from measurements of degree of coherence?" Proc. Phys. Soc. London 80, 1269-1272 (1962).

12. Lord Rayleigh (J. W. Strutt), "On the interference bands of approximately homogeneous light; in a letter to Prof. A. Michelson," Philos. Mag. 34, 407-411 (1892).

13. R. W. Gerchberg and W. O. Saxton, "A practical algorithm for the determination of phase from image and diffraction plane pictures," Optik (Stuttgart) 35, 237-246 (1972).

14. J. R. Fienup, "Phase retrieval algorithms: a comparison," Appl. Opt. 21, 2758-2769 (1982).

15. D. C. Youla and H. Webb, "Image restoration by the method of convex projections: Part I-theory," IEEE Trans. Med. Imaging MI-1, 81-94 (1982).

16. A. Lent and H. Tuy, "An iterative method for the extrapolation of band-limited functions," J. Math. Anal. Appl. 83, 554-565 (1981).

17. A. Levi and H. Stark, "Signal reconstruction from phase by projection onto convex sets," J. Opt. Soc. Am. 73, 810-822 (1983).

18. H. J. Trussell and M. R. Civanlar, "The feasible solution in signal restoration," IEEE Trans. Acoust. Speech Signal Process. ASP-32, 201-212 (1984)

19. L. M. Brègman, "The method of successive projection for finding a common point of convex sets," Sov. Math. Dokl. 6, 688-692 (1965).

20. P. L. Combettes, "The foundations of set theoretic estimation," Proc. IEEE 81, 182-208 (1993).

21. P. L. Combettes, "The convex feasibility problem in image recovery," in Advances in Imaging and Electron Physics, P. W. Hawkes, ed. (Academic, Orlando, Fla., 1996), Vol. 95, pp. $155-270$.

22. H. Stark, ed., Image Recovery: Theory and Application (Academic, Orlando, Fla., 1987)

23. H. Stark and Y. Yang, Vector Space Projections: A Numerical Approach to Signal and Image Processing, Neural Nets, and Optics (Wiley, New York, 1998).

24. P. L. Combettes and H. J. Trussell, "Stability of the linear prediction filter: a set theoretic approach," in Proceedings of the IEEE International Conference on Acoustics, Speech, and Signal Processing (Institute of Electrical and Electronics Engineers, New York, 1988), pp. 2288-2291.

25. M. Hedley, H. Yan, and D. Rosenfeld, "Motion artifact correction in MRI using generalized projections," IEEE Trans. Med. Imaging 10, 40-46 (1991).

26. R. G. Hoptroff, P. W. McOwan, T. J. Hall, W. J. Hossak, and R. E. Burge, "Two optimization approaches to cohoe design," Opt. Commun. 73, 188-194 (1989).

27. B. E. A. Saleh and K. M. Nashold, "Image construction: optimum amplitude and phase masks in photolithography," Appl. Opt. 24, 1432-1437 (1985).

28. A. Levi and H. Stark, "Image restoration by the method of generalized projections with application to restoration from magnitude," J. Opt. Soc. Am. A 1, 932-943 (1984).

29. A. Levi and H. Stark, "Restoration from phase and magnitude by generalized projections," in Image Recovery: Theory and Applications, H. Stark, ed. (Academic, Orlando, Fla., 1987), pp 277-320.

30. J. A. Cadzow, "Signal enhancement-a composite property mapping algorithm," IEEE Trans. Acoust. Speech Signal Process. 36, 49-62 (1988).

31. P. L. Combettes and H. J. Trussell, "Method of successive projections for finding a common point of sets in metric spaces," J. Optim. Theory Appl. 67, 487-507 (1990)

32. N. E. Hurt, "Signal enhancement and the method of successive projections," Acta Appl. Math. 23, 145-162 (1991).

33. S. Chrétien and P. Bondon, "Cyclic projection methods on a class of nonconvex sets," Numer. Funct. Anal. Optim. 17, 37-56 (1996)

34. R. Barakat and G. Newsam, "Algorithms for reconstruction of partially known, band-limited Fourier-transform pairs from noisy data," J. Opt. Soc. Am. A 2, 2027-2039 (1985).

35. R. Barakat and G. Newsam, "Algorithms for reconstruction of partially known, band-limited Fourier-transform pairs from noisy data. II. The nonlinear problem of phase retrieval," J. Integral Eq. 9, 77-125 (1985).

36. The issue of nonuniqueness of the projection is not to be confused with the uniqueness of solutions to the phase problem. The results surveyed, for instance, in Ref. 37, are not affected by the multivaluedness of the projection operators.

37. M. H. Hayes, "The unique reconstruction of multidimensional sequences from Fourier transform magnitude or phase," in Image Recovery: Theory and Application, $\mathrm{H}$. Stark, ed. (Academic, Orlando, Fla., 1987), pp. 195-230.

38. D. R. Luke, "Analysis of wavefront reconstruction and deconvolution in adaptive optics," Ph.D. thesis (University of Washington, Seattle, Wash., June 2001), ftp:// amath.washington.edu/pub/russell/Dissertation.ps.gz.

39. D. R. Luke, J. V. Burke, and R. Lyon, "Optical wavefront reconstruction: theory and numerical methods," SIAM Rev. 44(2) (2002), ftp://amath.washington.edu/pub/russell/ Luke_Burke_Lyon_01.ps.gz.

40. J. R. Fienup, "Reconstruction of an object from the modulus of its Fourier transform," Opt. Lett. 3, 27-29 (1978).

41. J. R. Fienup, "Space object imaging through the turbulent atmosphere," Opt. Eng. 18, 529-534 (1979).

42. J. R. Fienup, "Iterative method applied to image reconstruction and to computer-generated holograms," Opt. Eng. 19, 297-305 (1980).

43. J. W. Goodman, Statistical Optics (Wiley Interscience, New York, 1985)

44. "a.e." stands for "almost everywhere" in the sense of measure theory, since, strictly speaking, the elements of $\mathcal{L}$ are classes of equivalence of signals that may differ on a set of zero measure. For technical details on $\mathcal{L}$, see, for instance, Ref. 45.

45. C. D. Aliprantis and K. C. Border, Infinite-Dimensional Analysis, 2nd ed. (Springer-Verlag, Berlin, 1999).

46. J. N. Cederquist, J. R. Fienup, C. C. Wackerman, S. R. Robinson, and D. Kryskowski, "Wave-front phase estimation from Fourier intensity measurements," J. Opt. Soc. Am. A 6, 1020-1026 (1989)

47. P. L. Combettes, "Inconsistent signal feasibility problems: least-squares solutions in a product space," IEEE Trans. Signal Process. 42, 2955-2966 (1994). 
48. G. T. Herman, Image Reconstruction from Projections-The Fundamentals of Computerized Tomography (Academic, New York, 1980)

49. J. H. Seldin and J. R. Fienup, "Numerical investigation of the uniqueness of phase retrieval," J. Opt. Soc. Am. A 7, 412-427 (1990).

50. H. H. Bauschke, "The composition of finitely many projections onto closed convex sets in Hilbert space is asymptotically regular,” Proc. Am. Math. Soc. (to be published).

51. H. H. Bauschke and J. M. Borwein, "On the convergence of von Neumann's alternating projection algorithm for two sets," Set-Valued Anal. 1, 185-212 (1993).

52. H. H. Bauschke, J. M. Borwein, and A. S. Lewis, "The method of cyclic projections for closed convex sets in Hilbert space," in Recent Developments in Optimization Theory and Nonlinear Analysis (American Mathematical Society, Providence, R.I., 1997), pp. 1-38.

53. P. L. Combettes and P. Bondon, "Hard-constrained inconsistent signal feasibility problems," IEEE Trans. Signal Process. 47, 2460-2468 (1999).

54. L. G. Gubin, B. T. Polyak, and E. V. Raik, "The method of projections for finding the common point of convex sets," USSR Comput. Math. Math. Phys. 7, 1-24 (1967).

55. D. C. Youla and V. Velasco, "Extensions of a result on the synthesis of signals in the presence of inconsistent constraints," IEEE Trans. Circuits Syst. CAS-33, 465-468 (1986).

56. Iterative signal recovery projection algorithms have also been implemented optically without sampling the continuous waveforms (e.g., Ref. 57). In such instances, the underlying signal space is $\mathcal{L}$ itself.

57. R. J. Marks II, "Coherent optical extrapolation of 2-D bandlimited signals: processor theory," Appl. Opt. 19, 1670$1672(1980)$

58. Let $R$ be a set of real numbers. If $R \neq \varnothing$, then $\inf (R)$ stands for the infimum of $R$, i.e., the largest number in [ $-\infty$, $+\infty]$ that is smaller than or equal to all elements of $R$. By convention, $\inf (\varnothing)=+\infty$

59. J.-P. Aubin and H. Frankowska, Set-Valued Analysis (Birkhäuser, Boston, Mass., 1990).

60. For theoretical reasons, the sets (and functions) that we deal with must be "measurable"- this is not the same as being "physically measurable" or "observable"! For our purposes, measurable sets and functions constitute a sufficiently large class to work with; thus all closed and open subsets (and all continuous functions) are measurable, as well as various combinations of those.

61. Mathematically, this set is assumed to have nonzero measure.

62. The complex Hilbert space $\mathcal{L}$ from the phase retrieval problem is also a real Hilbert space, provided that we use the real part of the inner product as the new inner product.

63. C. Tisseron, Notions de Topologie-Introduction aux Espaces Fonctionnels (Hermann, Paris, 1985).

64. Recall the notation from Remark 3.4

65. F. Deutsch, Best Approximation in Inner Product Spaces (Springer-Verlag, New York, 2001).

66. In our setting, this means that the set $\left\{t \in \mathbb{R}^{N}: A(t) \cap Z\right.$ $\neq \varnothing\}$ is measurable for every closed (or, equivalently, open) set $Z$ in C; see Section 8.1 in Ref. 59 and Section 14.A in Ref. 67.

67. R. T. Rockafellar and R. J.-B. Wets, Variational Analysis (Springer-Verlag, Berlin, 1998).

68. If $x \in \mathcal{L}$, then $\operatorname{Re}(x)$ denotes the function defined by $\operatorname{Re}(x)$ : $t \mapsto \operatorname{Re}[x(t)]$, and $[\operatorname{Re}(x)]^{+}$subsequently denotes the positive part: $[\operatorname{Re}(x)]^{+}: t \mapsto \max \{0, \operatorname{Re}[x(t)]\}$.

69. R. W. Gerchberg, "Super-resolution through error energy reduction," Opt. Acta 21, 709-720 (1974).

70. D. C. Youla, "Generalized image restoration by the method of alternating orthogonal projections," IEEE Trans. Circuits Syst. CAS-25, 694-702 (1978)

71. A. Papoulis, "A new algorithm in spectral analysis and band-limited extrapolation," IEEE Trans. Circuits Syst. CAS-22, 735-742 (1975).
72. A. E. Çetin and R. Ansari, "Convolution-based framework for signal recovery and applications," J. Opt. Soc. Am. A 5, 1193-1200 (1988)

73. K. Goebel and W. A. Kirk, Topics in Metric Fixed Point Theory (Cambridge U. Press, Cambridge, UK, 1990).

74. Z. Opial, "Weak convergence of the sequence of successive approximations for nonexpansive mappings," Bull. Am. Math. Soc. 73, 591-597 (1967).

75. A. Genel and J. Lindenstrauss, "An example concerning fixed points," Isr. J. Math. 22, 81-86 (1975).

76. Alternative descriptions of these algorithms have been proposed; see, for instance, Ref. 77.

77. G. T. Herman and D.-W. Ro, "Image recovery using iterative data refinement with relaxation," Opt. Eng. 29, 513-523 (1990).

78. Another formulation of the algorithm also includes a nonnegativity constraint. ${ }^{14}$

79. The reason for this difference is that Fienup, on page 2763 of Ref. 14, defines $\gamma$ as the set of all points where (in our notation) $P_{M}\left(x_{n}\right)$ violates the object domain constraints. Hence $\gamma=\left\{t \in\left[D:\left(P_{M}\left(x_{n}\right)\right)(t) \neq 0\right\}\right.$, or $t \in \gamma$ if and only if $t \in D$ and $P_{M}\left(x_{n}\right)(t) \neq 0$. It follows that $t$ belongs to the complement of $\gamma$ if and only if $t \in D$ or $P_{M}\left(x_{n}\right)(t)=0$. The latter condition then leads to this different interpretation of the HIO algorithm. Sticking with this interpretation for another moment, we could set $D(n)=D \cup\{t$ $\left.\in \mathcal{C} D: P_{M}\left(x_{n}\right)(t)=0\right\} \quad$ and $\quad S(n)=\left\{z \in \mathcal{L}: z \cdot 1_{C D(n)}\right.$ $=0\}$ and obtain analogously

$$
x_{n+1}=\left(P_{S(n)}\left(2 P_{M}-I\right)+\left(I-P_{M}\right)\right)\left(x_{n}\right) .
$$

In practical experiments for problem (5), however, this ambiguity has hardly an impact, as the sets $\gamma$ and $C D$ almost always coincide.

80. H. Takajo, T. Shizuma, T. Takahashi, and S. Takahata, "Reconstruction of an object from its noisy Fourier modulus: ideal estimate of the object to be constructed and a method that attempts to find that object," Appl. Opt. 38, 5568-5576 (1999).

81. H. Takajo, T. Takahashi, and T. Shizuma, "Further study on the convergence property of the hybrid input-output algorithm used for phase retrieval," J. Opt. Soc. Am. A 16, 2163-2168 (1999).

82. H. Takajo, T. Takahashi, R. Ueda, and M. Taninaka, "Study on the convergence property of the hybrid input-output algorithm used for phase retrieval," J. Opt. Soc. Am. A 15, 2849-2861 (1998)

83. The corresponding mask is certainly much easier to implement.

84. J. R. Fienup and C. C. Wackerman, "Phase retrieval stagnation problems and solutions," J. Opt. Soc. Am. A 3, $1897-$ 1907 (1986).

85. The algorithms discussed here for solving problem (25) can be viewed in the broader context of finding a zero of the sum of two maximal monotone operators. Good starting points are Refs. 86-88.

86. P. L. Combettes, "Fejér-monotonicity in convex optimization," in Encyclopedia of Optimization, C. A. Floudas and P. M. Pardalos, eds. (Kluwer, New York, 2001), Vol. 2, pp. 106114.

87. J. Eckstein, "Splitting methods for monotone operators with applications to parallel optimization," Ph.D. thesis (Department of Civil Engineering, Massachusetts Institute of Technology, Cambridge, Mass., 1989), available as Rep. LIDS-TH-1877 (Laboratory for Information and Decision Sciences, MIT).

88. J. Eckstein and D. P. Bertsekas, "On the Douglas-Rachford splitting method and the proximal point algorithm for maximal monotone operators," Math. Program. (Ser. A) 55, 293-318 (1992).

89. Unfortunately, $P_{A} P_{B}$ is generally not firmly nonexpansive. However, it is strongly nonexpansive (see Ref. 90 for a precise definition and further information), and, for this class of mappings, a result corresponding to Fact 3.20 does exist. $^{90}$ 
90. R. E. Bruck and S. Reich, "Nonexpansive projections and resolvents of accretive operators in Banach spaces," Houston J. Math. 3, 459-470 (1977).

91. H. H. Bauschke and J. M. Borwein, "On projection algorithms for solving convex feasibility problems," SIAM Rev. 38, 367-426 (1996).

92. H. H. Bauschke, "Projection algorithms: results and open problems," in Inherently Parallel Algorithms in Feasibility and Optimization and Their Applications, Studies in Computational Mathematics, D. Butnariu, Y. Censor, and S. Reich, eds. (Elsevier, Amsterdam, 2001), Vol. 8, pp. 11-22.

93. P. L. Combettes, "Quasi-Fejérian analysis of some optimization algorithms," in Inherently Parallel Algorithms in Feasibility and Optimization and Their Applications, Studies in Computational Mathematics, D. Butnariu, Y. Censor, and S. Reich, eds. (Elsevier, Amsterdam, 2001), Vol. 8, pp. 115152.

94. R. L. Dykstra, "An algorithm for restricted least squares regression,” J. Am. Stat. Assoc. 78, 837-842 (1983).

95. J. P. Boyle and R. L. Dykstra, "A method for finding projections onto the intersection of convex sets in Hilbert spaces," in Advances in Order Restricted Statistical Inference (Springer, Berlin, 1986), pp. 28-47.

96. In the aforementioned context of maximal monotone operators, ${ }^{85}$ Dykstra's algorithm can be interpreted as a tight version of the Peaceman-Rachford algorithm. See page 77 in Ref. 87 for further information. Let us also note that in the standard linear case, the Peaceman-Rachford and Douglas-Rachford algorithms can be viewed from a unifying standpoint (see Section 7.4 in Ref. 97).

97. R. S. Varga, Matrix Iterative Analysis, 2nd ed. (SpringerVerlag, New York, 2000).

98. H. H. Bauschke and J. M. Borwein, "Dykstra's alternating projection algorithm for two sets," J. Approx. Theory 79, 418-443 (1994).

99. H. H. Bauschke and A. S. Lewis, "Dykstra's algorithm with Bregman projections: a convergence proof," Optimization 48, 409-427 (2000).

100. N. Gaffke and R. Mathar, "A cyclic projection algorithm via duality," Metrika 36, 29-54 (1989).

101. S.-P. Han, "A successive projection method," Math. Program. (Ser. A) 40, 1-14 (1988).

102. H. Hundal and F. Deutsch, "Two generalizations of Dykstra's cyclic projections algorithm," Math. Program. (Ser. A) 77, 335-355 (1997).
103. P. L. Combettes, "Signal recovery by best feasible approximation," IEEE Trans. Image Process. 2, 269-271 (1993).

104. The Douglas-Rachford algorithm was originally developed as a linear implicit iterative method to solve partial differential equations in Ref. 105 (see also Chap. 7 in Ref. 97). It was extended to an operator splitting method for finding a zero of the sum of two maximal monotone operators by $\mathrm{Li}$ ons and Mercier in Ref. 106. When it is applied to the normal cone maps of the constraint sets, one obtains a method for solving problem (25). See Refs. 86-88 and 106 for further information.

105. J. Douglas and H. H. Rachford, "On the numerical solution of heat conduction problems in two or three space variables," Trans. Am. Math. Soc. 82, 421-439 (1956).

106. P.-L. Lions and B. Mercier, "Splitting algorithms for the sum of two nonlinear operators," SIAM (Soc. Ind. Appl. Math.) J. Numer. Anal. 16, 964-979 (1979).

107. $u$ is a weak cluster point of a sequence $\left(u_{n}\right)$ if there exists a subsequence $\left(u_{k_{n}}\right)$ such that $u_{k_{n}} \rightarrow u$.

108. If we had used the literal update rule for the HIO algorithm, the present observation would change only in one respect: the set $A$ would be replaced with $S(n)$ (see Remark 4.1 and Ref. 79) and hence vary with $n$.

109. E. H. Zarantonello, "Projections on convex sets in Hilbert space and spectral theory," in Contributions to Nonlinear Functional Analysis, E. H. Zarantonello, ed. (Academic, New York, 1971), pp. 237-424.

110. L. Debnath and P. Mikusiński, Introduction to Hilbert Spaces with Applications, 2nd ed. (Academic, San Diego, Calif., 1999).

111. However, as shown in Property 4.1 in Ref. 39, the set $M$ is not weakly closed; i.e., if a sequence $\left(x_{n}\right)$ of points in $M$ converges weakly to a point $x$, then $x$ may not be in $M$.

112. While $P_{B}$ is nonexpansive and therefore Lipschitz continuous, this property is not sufficient to draw the conclusion advertised in Corollary 6.1 in Ref. 88, namely (in our context), that $\left(P_{B} x_{n}\right)$ converges weakly to a point in $A \cap B$. Such a conclusion requires additional assumptions, e.g., that $P_{B}$ be weakly continuous (if so, then $P_{B} x_{n} \rightarrow P_{B} x$ ), as is the case when $\operatorname{dim} \mathcal{H}<+\infty$ (or when $B$ is a closed affine subspace). Note, however, that the projector onto a closed convex set may fail to be weakly continuous. An example is on page 245 in Ref. 109 . 